\title{
Detection of circulating colorectal cancer cells by a custom microfluid system before and after endoscopic metallic stent placement
}

\author{
REI ISHIBASHI ${ }^{1}$, SHUNTARO YOSHIDA ${ }^{1}$, NARIAKI ODAWARA ${ }^{1}$, TAKAHIRO KISHIKAWA $^{1}$, RYO KONDO $^{1}$, \\ AYAKO NAKADA $^{1}$, RYUNOSUKE HAKUTA ${ }^{1}$, NAMINATSU TAKAHARA ${ }^{1}$, ERI TANAKA ${ }^{1}$, KAZUMA SEKIBA ${ }^{1}$, \\ TAKAHIRO SEIMIYA ${ }^{1}$, TAKASHI OHNAGA ${ }^{2}$, MOTOYUKI OTSUKA ${ }^{1}$ and KAZUHIKO KOIKE ${ }^{1}$ \\ ${ }^{1}$ Department of Gastroenterology, Graduate School of Medicine, The University of Tokyo, Tokyo 113-8655; \\ ${ }^{2}$ Central Research Institute, Toyama Industrial Technology Center, Toyama 933-0981, Japan
}

Received July 10, 2019; Accepted August 30, 2019

DOI: $10.3892 / \mathrm{ol} .2019 .11047$

\begin{abstract}
Although the detection of circulating tumor cells (CTCs) should be crucial for future personalized medicine, no efficient and flexible methods have been established. The current study established a polymeric custom-made chip for capturing CTCs with a high efficiency and flexibility. As an example of clinical application, the effects of self-expandable metallic stent (SEMS) placement on the release of cancer cells into the blood of patients with colorectal cancer and bowel obstruction were analyzed. This was assessed as the placement of SEMS may cause mechanical damage and physical force to malignant tissue, increasing the risk of cancer cell release into the bloodstream. The present study examined the number of CTCs using a custom-made chip, before, at $24 \mathrm{~h}$ after and at 4 days after SEMS placement in patients with colorectal cancer. The results revealed that, among the 13 patients examined, the number of CTCs was increased in three cases at $24 \mathrm{~h}$ after SEMS placement. However, this increase was temporary. The number of CTCs also decreased at 4 days after stent placement in most cases. The CTC chip of the current study detected the number of CD133-positive cancer stem-like cells, which did not change, even in the patient whose total number of CTCs temporarily increased. The results indicated that this custom-made microfluid system can efficiently and flexibly detect CTCs, demonstrating its potential for obtaining information during the management of patients with cancer.
\end{abstract}

Correspondence to: Dr Motoyuki Otsuka, Department of Gastroenterology, Graduate School of Medicine, The University of Tokyo, 5-3-1 Hongo, Bunkyo-ku, Tokyo 113-8655, Japan

E-mail: otsukamo-tky@umin.ac.jp

Abbreviations: CRC, colorectal cancer; CTC, circulating tumor cell; SEMS, self-expandable metallic stent; cfDNA, cell-free DNA

Key words: circulating tumor cells, colorectal cancer, CD133, cancer stem-like cell, self-expandable metallic stents

\section{Introduction}

Circulating tumor cells (CTCs) are potential surrogates for distant metastasis and promising novel biomarkers for malignancies (1). However, the detection of rare tumor-derived cells among a majority of normal hematological cells still remains technically challenging, while diverse methods have been reported (2-4). We recently established a novel microfluid system to capture CTCs (CTC chip), which has the advantages of convenience, efficacy, and flexibility $(2,5)$.

Colorectal cancer (CRC) is the third most common diagnosed cancer in males and the second in females; it caused 83,200 deaths globally in 2015 (6). At the time of diagnosis, $10-30 \%$ of patients with CRC have acute large bowel obstruction $(7,8)$. Emergent decompression is necessary for large bowel obstruction, and self-expandable metallic stent (SEMS) placement is a clinical choice as a minimally invasive non-surgical treatment. With accumulating experience and progress in equipment development, SEMS placement has positive short-term outcomes, and technical and clinical success rates of $91-98$ and $89-92 \%$, respectively, have been reported $(8,9)$.

Although the efficacy and feasibility of endoscopic SEMS placement in obstructive CRC are well-documented, several clinical concerns regarding SEMS placement remain $(10,11)$. One of the major concerns is the risk of increased cancer cells in the bloodstream due to mechanical damage and pressure applied to the tissues by SEMS placement, possibly leading to increased metastasis $(12,13)$. In fact, circulating tumor-derived DNA levels are elevated following SEMS placement, likely due to mechanical damage (14). Therefore, it is hypothesized that cancer cells are also released by mechanical damage to tissues during SEMS placement.

In this study, as a trial of clinical application of our microfluid system to capture CTCs, we examined differences in CTC levels before and after SEMS placement in patients with obstructive CRC using our custom CTC chip to evaluate the risk of increasing the quantity of cancer cells in the blood by SEMS placement. Additionally, based on the recent evidence that cancer stem cells released from primary lesions 
in the blood play a central role in establishing metastasis in distant organs (15), we examined changes in the number of CD133-positive CTCs, CRC stem-like cell markers $(16,17)$, to better show the flexibility of the custom CTC chip.

\section{Materials and methods}

Study approval. This prospective single-center observational study was conducted at the University of Tokyo Hospital. We examined differences in the numbers of CTCs before and after endoscopic SEMS placement for obstructive CRC. We included thirteen patients with primary CRC with obstruction and performed SEMS placement between July 2017 and April 2019 at the University of Tokyo Hospital. The study protocol was approved by the Institutional Review Board of the University of Tokyo Hospital (approved no. 11557), and written informed consent was obtained from all participating patients before enrollment in this study. The study was carried out in accordance with the relevant guidelines.

CTC chip preparation. Polymeric CTC chips consist of two types of micropole to increase capture efficacy $(2,5)$. To capture CTCs, a two-step coating of CTC chip micropoles and surfaces with antibodies was performed. First, the CTC chip was coated with goat anti-rabbit immunoglobulin $\mathrm{G}$ antibodies (\#SAB3700970; Sigma-Aldrich; Merck KGaA), which were diluted 1:20 and incubated for more than $3 \mathrm{~h}$ at $4^{\circ} \mathrm{C}$. Next, the chip was incubated with secondary antibodies, rabbit anti-human EpCAM antibodies (\#36746; Cell Signaling Technology, Inc.), which were diluted 1:100 and incubated for $1 \mathrm{~h}$ at room temperature. CTC chips were coated with antibodies on the day before each SEMS placement.

Sample collection and CTC chip processing. Peripheral blood samples $(5 \mathrm{ml})$ were collected into tubes containing ethylenediaminetetraacetic acid before SEMS placement, $24 \mathrm{~h}$ after SEMS placement, and 4 days after SEMS placement. Within $12 \mathrm{~h}$ of collection, samples were centrifuged at $300 \mathrm{x} \mathrm{g}$ for $5 \mathrm{~min}$ at $4^{\circ} \mathrm{C}$. After separating the plasma, we collected $1.5 \mathrm{ml}$ mixed buffy coat and red blood cells as a processed sample (5). Samples were transferred into the prepared CTC-chip microfluidic system using an automated syringe pump. Briefly, processed samples $(1.5 \mathrm{ml})$ were applied to the CTC chip using a syringe pump at a constant flow rate of $1.0 \mathrm{ml} / \mathrm{h}$. The CTC chip was then rinsed with $10 \%$ bovine serum albumin in PBS three times at the same flow rate. After CTC capture, immunocytochemistry was performed. For preliminary experiments using cell lines with the CTC chip, approximately $1 \times 10^{4}$ cells were dissolved in $1.5 \mathrm{ml}$ PBS and applied to the chip.

Immunocytochemistry of CTC chips. After CTC capture, immunostaining of the CTCs was performed using human CK19 antibodies, as reported previously (5), and, in two cases, using anti-CD133 antibodies. When using CD133 antibodies, before fixation with $4 \%$ paraformaldehyde in PBS, CTCs were incubated with mouse anti-human CD133 antibodies conjugated with Alexa Fluor 615 (\#130-113-671; Miltenyi Biotec $\mathrm{GmbH}$ ), which were diluted 1:50 for $10 \mathrm{~min}$ at room temperature in the CTC-chip microfluidic system. After fixation, CTCs were incubated with sheep anti-human CK19 antibodies (\#sc-33119; Santa Cruz Biotechnology), which were diluted 1:200 and incubated for $1 \mathrm{~h}$. Then, donkey anti-sheep antibodies conjugated with Alexa Fluor 488 (\#1807723; Thermo Fisher Scientific), which were diluted 1:1,000, were incubated for $30 \mathrm{~min}$ as secondary antibodies to visualize CTCs. Finally, the chip was covered with VectaShield with DAPI (Vector Laboratories). When preliminarily testing the compatibility of our CTC chips using cell lines, anti-CK19 antibodies and anti-CD45 antibodies were diluted 1:300, and the fluorescence was evaluated. The fluorescence signals were detected under an Olympus AX80 microscope.

Antibodies. The antibodies tested other than the CK19 and CD133 antibodies described above were as follows: Anti-wide cytokeratin antibodies (\#ab9377, Abcam; \#bs-1185R-A488, BIOSS Antibodies, Inc.), other hosts of anti-CK19 antibodies (\#sc-376126, \#sc-33119, \#AF3506, Santa Cruz Biotechnology), anti-Lgr5 (\#ab75732, Abcam; \#TA503316, OriGene; \#130-100-876, Miltenyi Biotec GmbH; \#21833-1-AP, Proteintech Group, Inc.), anti-human EpCAM (\#sc-25308, Santa Cruz Biotechnology; \#36746, Cell Signaling Technology), anti-HaloTag (G928A,Promega Corporation), and anti-CD45 (\#bs-05222R-A555, BIOSS; \#14579, Cell Signaling Technology; ab30446, Abcam; \#368520, BioLegend).

Cell culture. The human colon cancer cell line HCT116 and CD133-positive human pancreatic cancer cell line Capan-1 were obtained from the American Type Culture Collection. HCT116 and Capan-1 cells were maintained in McCoy's 5A media and Iscove's modified Dulbecco's medium, respectively, with $10 \%$ fetal bovine serum.

Plasmids and transfection. HaloTag-fused Lgr5-expressing plasmid was purchased from Promega. HCT116 cells were transiently transfected with the plasmid using the Effectene Transfection Reagent (Qiagen) according to the manufacturer's instructions. At $48 \mathrm{~h}$ post-transfection, cells were stained using anti-Lgr5 antibodies and anti-HaloTag antibodies to determine the suitability of the anti-Lgr5 antibodies for immunofluorescence cell staining.

Cell-free DNA quantitation. From separated plasmas of the patients' blood used for counting the number of CTCs, we collected cell-free DNA. Plasmas were centrifuged at $1,900 \mathrm{x} \mathrm{g}$ for $5 \mathrm{~min}$ at $4^{\circ} \mathrm{C}$. The supernatant were aspirated without disturbing the buffy coat layer. Aspirated plasmas were centrifuged at $16,000 \mathrm{x} \mathrm{g}$ for $10 \mathrm{~min}$ at $4^{\circ} \mathrm{C}$. From these samples, cell-free DNAs were collected using QIAamp Circulating Nucleic Acid Kit (Qiagen). Collected cell-free DNA samples were quantified with NanoDrop (Thermo Fisher Scientific).

Statistics. Statistically significant differences were identified using ANOVA and Bonferroni post-test. P-values <0.05 were considered to indicate statistical significance.

\section{Results}

Custom CTC chip efficiently detects CTCs in patient blood. To detect CTCs in patient blood, we used a custom-made polymeric CTC chip system, which was recently established (18). In the 
Table I. Compatibility of antibodies in the custom circulating tumor cell chip for the detection of epithelial cancer cell lines (HCT116; CK19 positive) and WBCs (CD45 positive).

\begin{tabular}{|c|c|c|c|c|}
\hline & $\begin{array}{l}\text { WideCK-Alexa488 } \\
\text { (BIOSS) }\end{array}$ & $\begin{array}{l}\text { WideCK-Alexa488 } \\
\text { (BIOSS) }\end{array}$ & $\begin{array}{l}\text { CK-19-Alexa488 } \\
\text { (Santa Cruz) }\end{array}$ & $\begin{array}{l}\text { WideCK-Alexa488 } \\
\text { (Abcam) }\end{array}$ \\
\hline Cell type & CD45-Alexa555 (BIOSS) & CD45-Alexa594 (CST) & CD45-Alexa594 (CST) & CD45-Alexa555 (Abcam) \\
\hline \multirow[t]{2}{*}{ HCT116 } & \pm & \pm & + & ++ \\
\hline & - & - & - & ++ \\
\hline \multirow[t]{2}{*}{ WBC } & - & - & - & Not determined \\
\hline & - & - & - & Not determined \\
\hline
\end{tabular}

-, negative staining; \pm , weak staining; + , positive staining; ++ , strongly positive staining; WBC, white blood cell; CST, Cell Signaling Technology; Santa Cruz, Santa Cruz Biotechnology, Inc.

first step, the chip surface was covered with base antibodies against capture antibodies (anti-EpCAM antibodies). In the next step, anti-EpCAM antibodies were trapped by the base antibodies. These antibodies are attached mainly to the micropoles on the chip surfaces. After centrifuging $5 \mathrm{ml}$ peripheral blood from a patient, only cell components, including buffy coat and red blood cells, were applied to the chip using an automatic syringe pump. After washing the chip with PBS using a syringe pump, we fixed the cells and performed immunocytochemistry using anti-CK19 antibodies to detect epithelial cells, which were recognized as CTCs (Fig. 1A). When needed, we used other antibodies in parallel to detect specific antigens with fluorescent secondary antibodies (Fig. 1A).

To establish the experimental settings, we tested several antibodies from different vendors to ensure efficient CTC detection by examining EpCAM-positive colon cancer cell line HCT116 cells or a mixture of HCT116 cells in human blood from a healthy volunteer. Although we used antibodies that were suitable for immunocytochemistry, their sensitivity differed significantly (Table I). In particular, anti-CD45 antibodies, which theoretically react with white blood cells, were not sufficiently reactive or were non-specifically reactive in our system (Table I). Because anti-CK19 antibodies reacted efficiently with HCT116 cells, we detected CTCs, which were captured by anti-EpCAM antibodies, by CK19 staining. Using this method, our CTC chip efficiently detected HCT116 cells, HCT116 cells mixed in blood, and CTCs from patients with cancer (Fig. 1B). The estimated capture efficiency was approximately $80 \%$, similar to our previous results (2).

The number of CTCs increases temporarily after endoscopic SEMS placement. As one example of clinical application of our CTC chip, we examined changes in the number of CTCs in patients who underwent endoscopic SEMS placement for obstructive CRC. We collected blood just before stenting, at $24 \mathrm{~h}$ after stenting, and at 4 days after stenting, and counted the CTCs. Typical CTCs from patients showed clusters (Fig. 2A), consistent with previous reports (19).

Among the 13 cases examined (Table II), CTCs were detected at some time point except in one patient (patient \#3). No obvious correlation between detectability and tumor stage, tumor differentiation grade, or tumor marker level was detected (Table II). At $24 \mathrm{~h}$ after stenting, three (patients \#1, \#4 and \#5) of the 11 patients in whom CTCs were measured at pre-stenting showed increased numbers of CTCs compared with those at the pre-stenting stage (Fig. 2B and Table II). However, the number of CTCs decreased at 4 days after stenting in all of these patients (Fig. 2B and Table II), suggesting that the increased number of CTCs after SEMS placement was temporary. Although three cases (patients \#2, \#7 and \#13) showed the largest numbers of CTCs at day 4 after SEMS placement, the degree of increase was only marginal (one or two CTCs), and CTC numbers did not differ significantly throughout the time course, as in the cases of patients \#6 and \#8, who had equal numbers of CTCs in their blood samples before and 4 days after stenting (Fig. 2B and Table II). The number of CTCs remained unchanged or decreased in two cases (patients \#9 and \#11) at 4 days after stenting compared with those at pre-stenting stage (Fig. 2B and Table II). The overall changes in CTC numbers at around the SEMS placement were not statistically significant. These results suggest that the number of CTCs may increase, but only temporarily, or may not significantly change, even after endoscopic SEMS placement.

CTC numbers and cell-free DNA concentration were not correlated. Cell-free DNA (cfDNA) is released from cancerous lesions and is a promising biomarker in the liquid biopsy field. To determine whether changes in CTC numbers and cfDNA were correlated, we determined the cfDNA concentration and the number of CTCs in six cases (patients \#1-6). Total cfDNA concentration, at around $10 \mathrm{ng} / \mathrm{ml}$, was not significantly changed by SEMS placement in this study (Fig. 2C and Table SI). Although the number of CTCs increased at $24 \mathrm{~h}$ after SEMS placement in patients \#1 and \#4, cfDNA concentration did not change significantly (Table SI), and the trend was not always correlated with changes in the number of CTCs (Table SI). From these results, it appears that CTCs and cfDNAs are released into the blood by different molecular mechanisms from cancerous lesions, and that SEMS placement does not have a significant impact on these phenomena.

Detection of CD133-positive cancer stem-like cells. Although CTCs are recognized as surrogates of distant metastasis, 
A
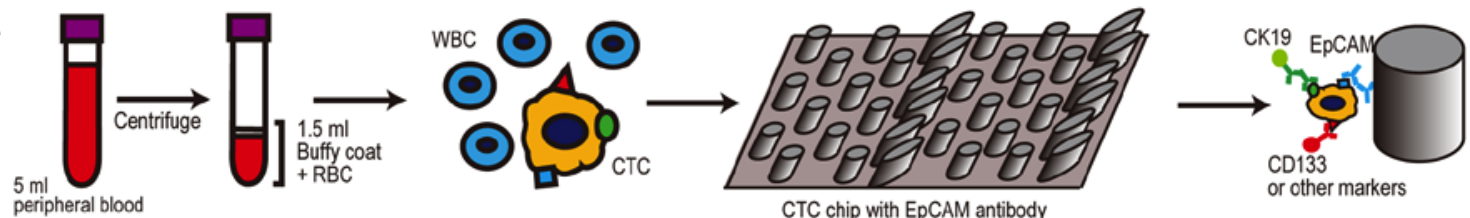

CTC chip with EpCAM antibody

B
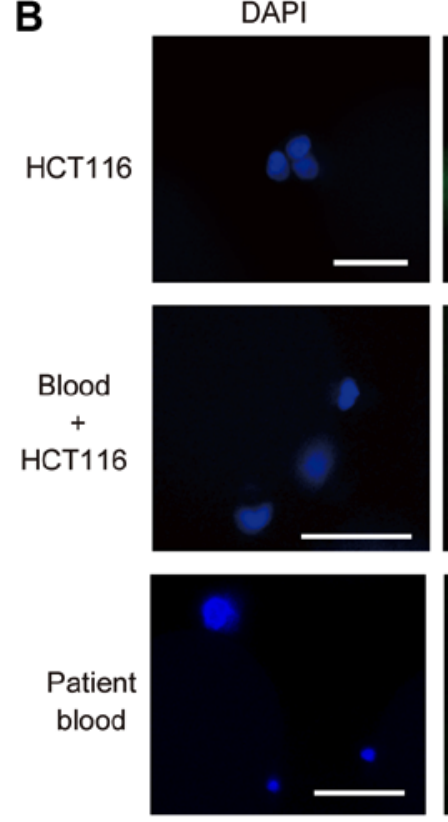
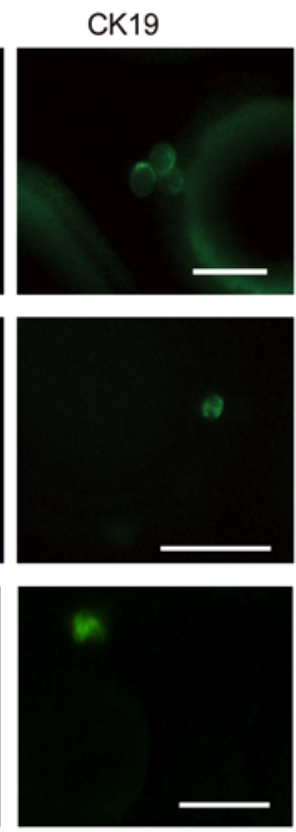
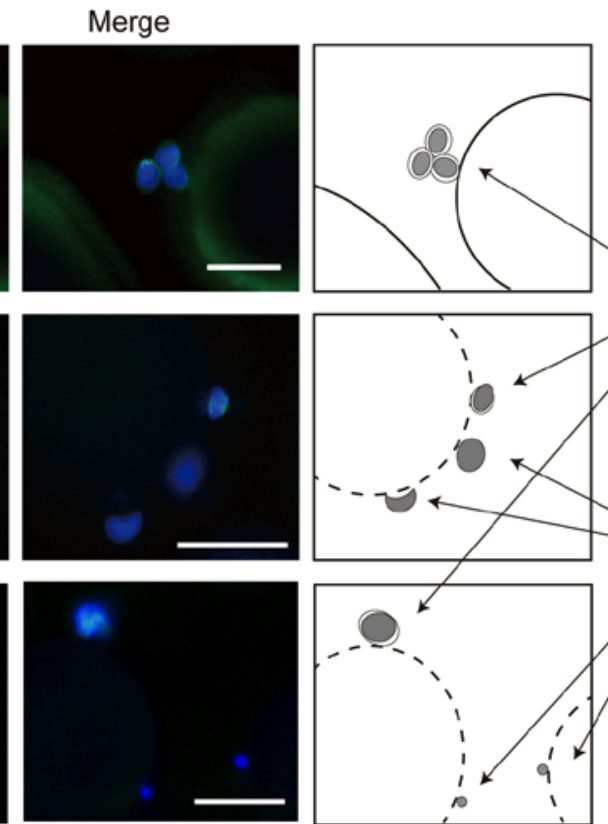
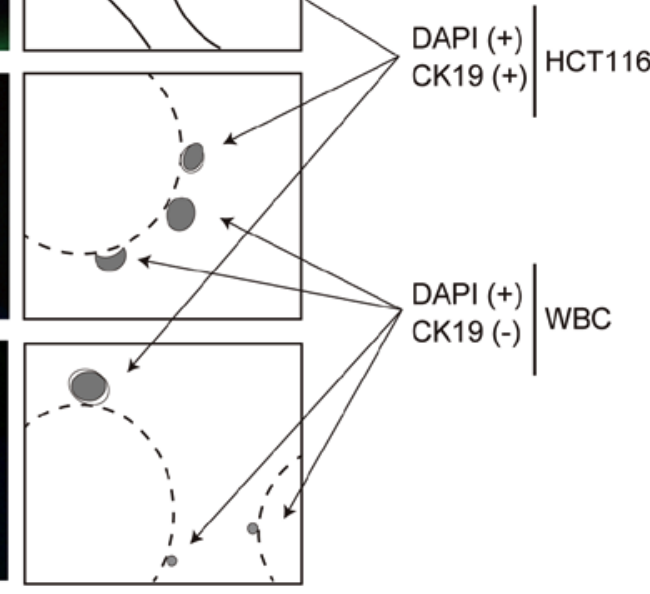

Figure 1. Capture of CTCs using the custom polymeric CTC chip. (A) Protocol for CTC capture with the custom CTC chip. Peripheral blood samples (5 ml) were collected from patients into tubes containing ethylenediaminetetraacetic acid. Samples were centrifuged at $300 \mathrm{x} g$ for 5 min at $4^{\circ} \mathrm{C}$. After removing plasma, cellular components including buffy coat were dissolved in $1.5 \mathrm{ml}$ PBS. Processed samples were transferred to the CTC chip using automatic syringes. Micropoles on the chip were coated with EpCAM antibodies to capture CTCs. Captured CTCs were stained with CK19 and CD133 or other antibodies. (B) Representative images of the captured CTCs and their schemes. HCT116 cells were captured (arrows) at the micropoles coated with EpCAM antibodies and the cells were stained with DAPI and CK19 antibodies (upper panels). HCT116 cells mixed with blood from a healthy donor were captured. All cells were stained with DAPI, but only HCT116 cells were stained with anti-CK19 (middle panels). CTCs in patient blood were captured. All cells were stained with DAPI, but only the CTCs were stained with anti-CK19 (lower panels). Scale bar, $50 \mu \mathrm{m}$. CTC, circulating tumor cells; WBC, white blood cell.

research has shown that not all CTCs have the potential to colonize distant organs. Because a recent report indicated that cancer stem-cells expressing Lgr5 are critical for the colonization of distant organs and the establishment of distant metastases (15), we attempted to stain for Lgr5 by immunocytochemistry, but were unsuccessful due to the lack of antibodies suitable for Lgr5 immunohistochemistry (Fig. S1). Alternatively, we searched for CD133-positive cancer stem-like cells among the CTCs detected in seven patients (Table II). Before examining the patient samples, we examined Capan-1 pancreatic cancer cell lines, some of which showed CD133 positivity. In fact, we detected some CD133-positive Capan-1 cells, and efficiently captured them (Fig. 3A).

Although the number of CTCs in patient \#7 was increased slightly at day 4 after SEMS placement, the number of CD133-positive cells was not increased $(3,2$, and 3 before, $24 \mathrm{~h}$ after, and 4 days after SEMS placement, respectively; Fig. 3B and Table II). In patient \#8, the total number of CTCs did not change, and the number of CD133-positive cells did not increase (2, 0 , and 2 before, $24 \mathrm{~h}$ after, and 4 days after SEMS placement, respectively). In other five cases except one case (patient \#13) in which one CD133-positive cell was detected only at 4 days after SEMS placement, CD133-positive cells did not increase at 4 days after stenting (Table II). Overall, the changes of the number of CD133-positive cells by SEMS placement were not statistically significant (Fig. 3C). Although the number of the cases tested is small, these results suggest that even in cases in which the number of CTCs increased after SEMS placement, cancer stem-like cells barely increased following stent placement, and such analyses could be done by the custom CTC chip.

\section{Discussion}

Because CTCs may reflect characteristics of primary cancer lesions, CTC detection will be crucial for personalized medicine, which will be implemented in the very near future. However, their detection remains challenging because convenient methods for CTC detection have not yet been established. In this study, we applied a novel microfluid system to determine CTC numbers around endoscopic SEMS placement, as a trial.

Although endoscopic decompression with an SEMS for obstructive malignant bowel obstruction is clinically effective, without severe invasiveness, and improves short-term outcomes $(20,21)$, its potential oncological safety is a concern due to the related risks of tumor dissemination by direct effects of 
A

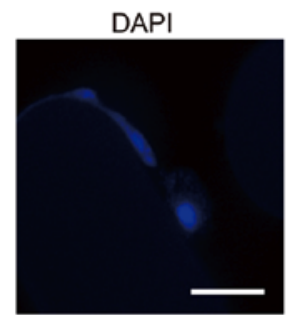

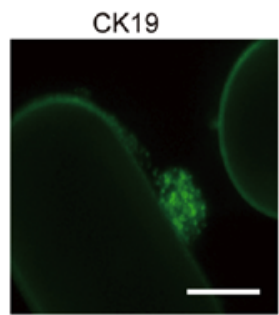
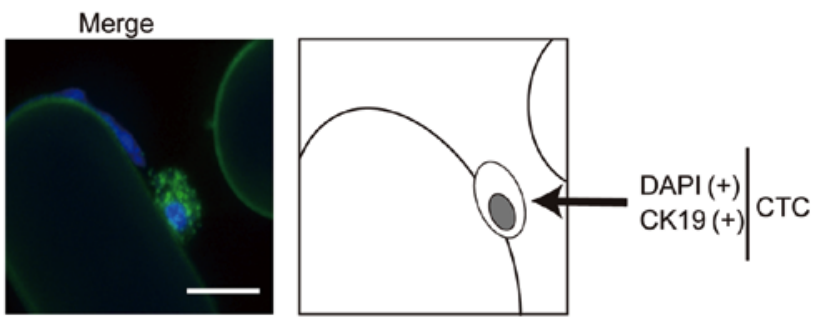

B

CTC number before and after stenting

n.s.

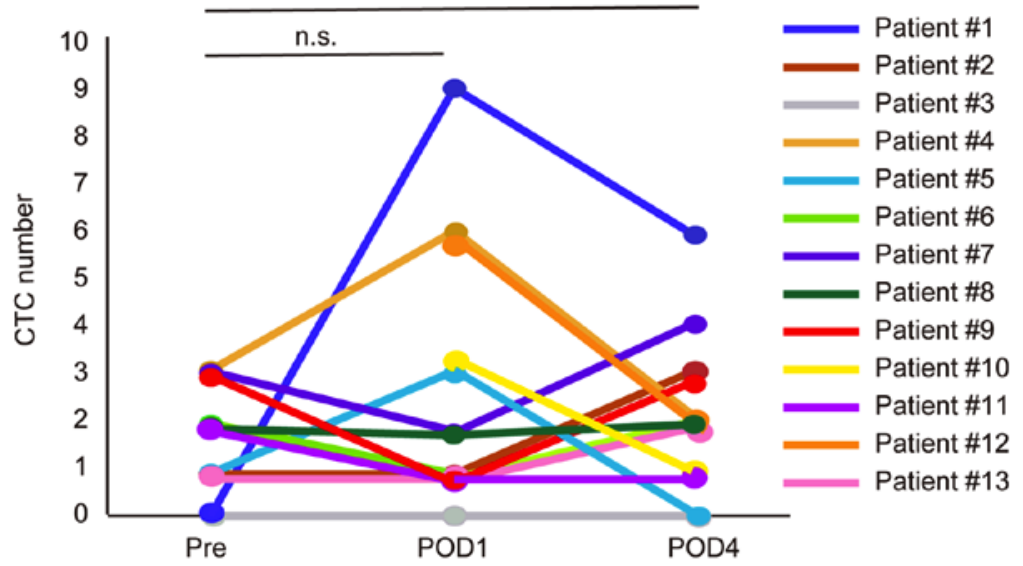

C

cfDNA concentration before and after stenting

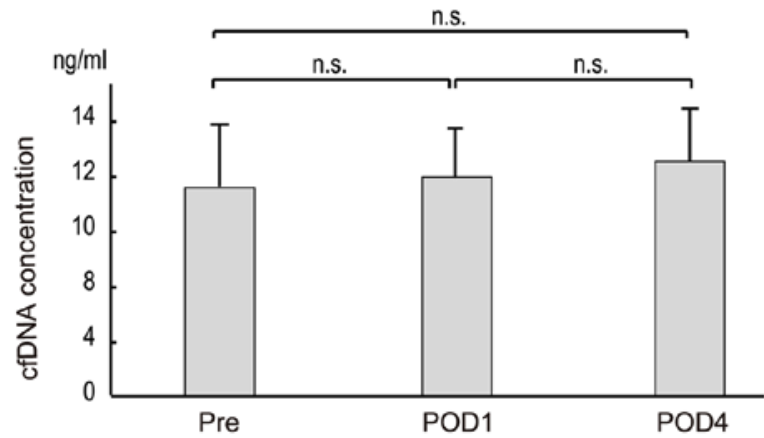

Figure 2. CTC number, CTCs and cfDNA concentrations following SEMS placement. (A) Fluorescence-stained CTCs captured by the CTC chip after SEMS placement. CTCs were stained with DAPI and CK19 (scale bar, $50 \mu \mathrm{m}$ ). (B) Differences in the numbers of CTCs before and after SEMS placement. The number of CTCs increased at $24 \mathrm{~h}$ after SEMS placement and decreased 4 days after SEMS placement in three cases (patient 1,4 and 5). The number of CTCs was slightly increased at 4 days after SEMS placement compared with those before SEMS placement in three cases (patient 2,7 and 13). The number of CTCs before SEMS placement was not tested in two cases (patient 10 and 12). In the remaining five cases (patient 3, 6, 8,9 and 11), CTC numbers remained unchanged or decreased. (C) cfDNA concentrations in plasma were determined in patients 1-6 before and after SEMS placement. Pre, before SEMS placement; POD1 and POD4, $24 \mathrm{~h}$ and 4 days after SEMS placement, respectively; n.s., no significance; CTC, circulating tumor cells; cfDNA, cell-free DNA; SEMS, self-expandable metallic stents.

physical forces on carcinoma tissue. However, we observed that endoscopic SEMS placement may temporarily increase CTC numbers, but does not drastically increase the number of cancer cells or significantly increase the release of cancer stem cells into the bloodstream. We found that the increase in the number of CTCs just after SEMS placement was temporary, as CTC levels tended to return rapidly to their former state, by day 4 after placement. These results suggest that the release of cancer cells into the blood by SEMS placement is due mainly to temporary compression related to aspects of the placement technique, such as the air supply and the initiation of physical force on the tissues, and the potential increased release of cancer cells into the blood does not significantly continue, due to SEMS placement itself.
The mechanism of the decrease in CTC numbers after the temporary increase caused by SEMS placement can be explained by two main hypotheses: That those cells cannot survive because they become trapped by immune surveillance or reticuloendothelial systems, or that those cells colonize distant organs. Recent reports have shown that not all CTCs have the potential for micrometastasis by the colonization of distant organs, but some specific CTCs have such features, which may be cancer stem-cell like features (22). In this study, we initially attempted to determine the existence of Lgr5-positive cancer cells, which were recently reported to be colonic stem cells (23), but because no antibody against Lgr5 was suitable for immunocytochemistry, despite testing of several antibodies, we alternatively tested for 


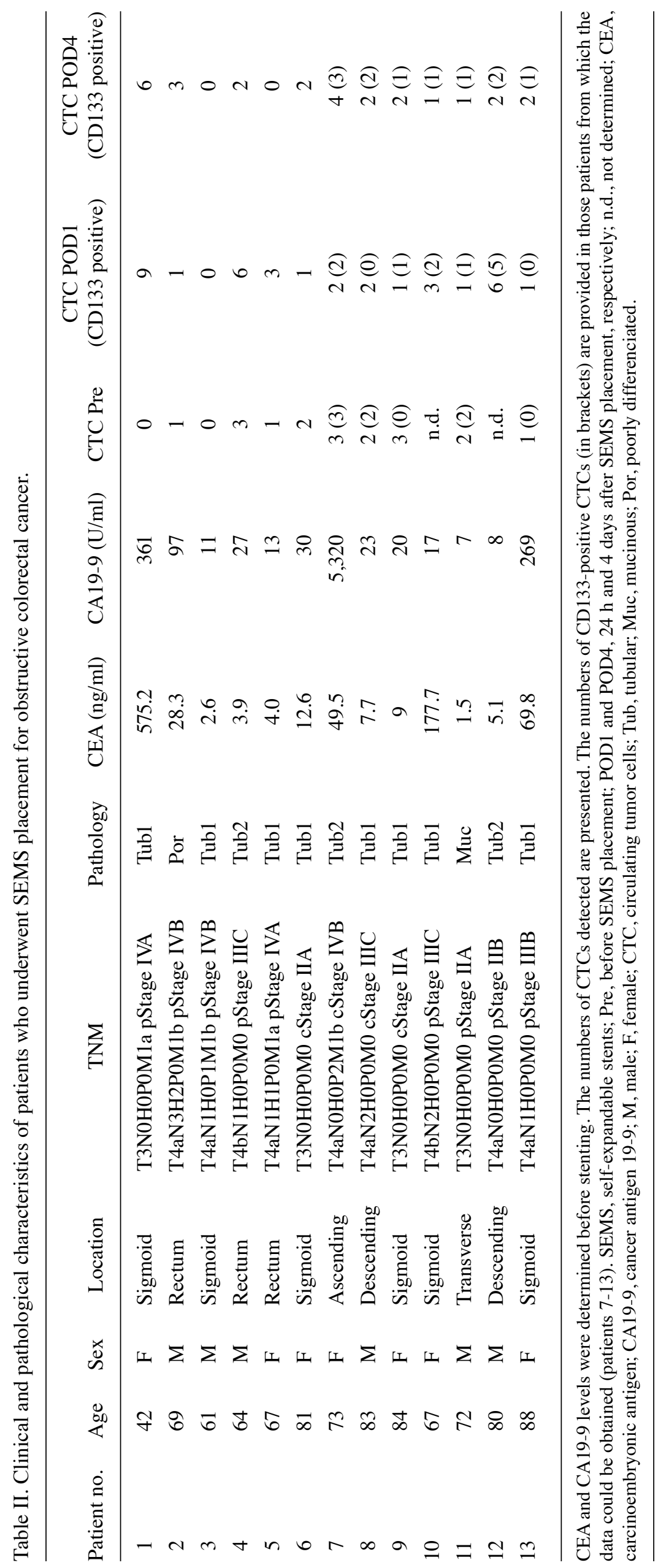


A

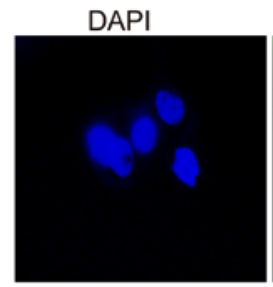

CK19

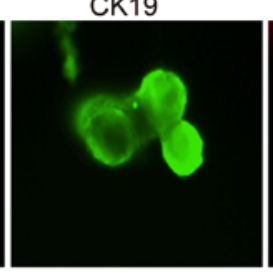

CD133
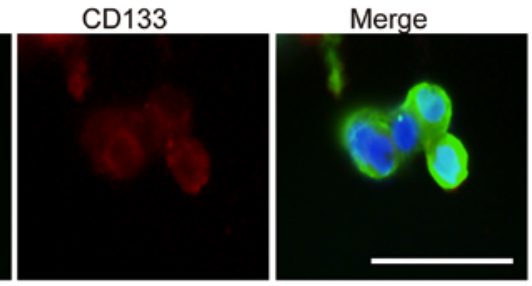

B
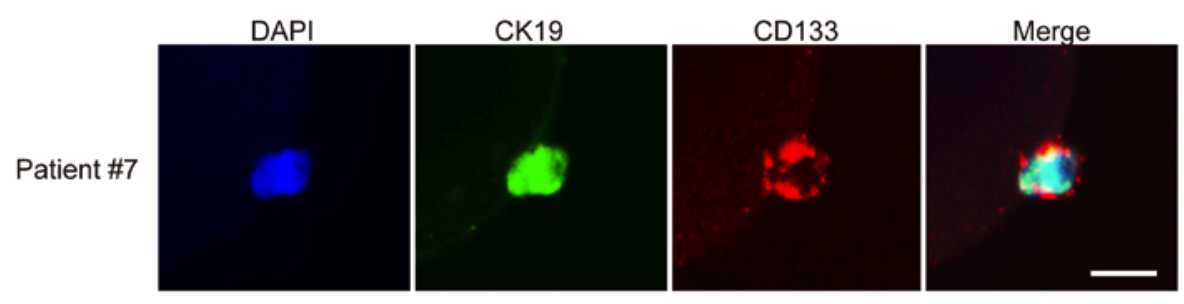

C

CD133-positive cells before and after stenting

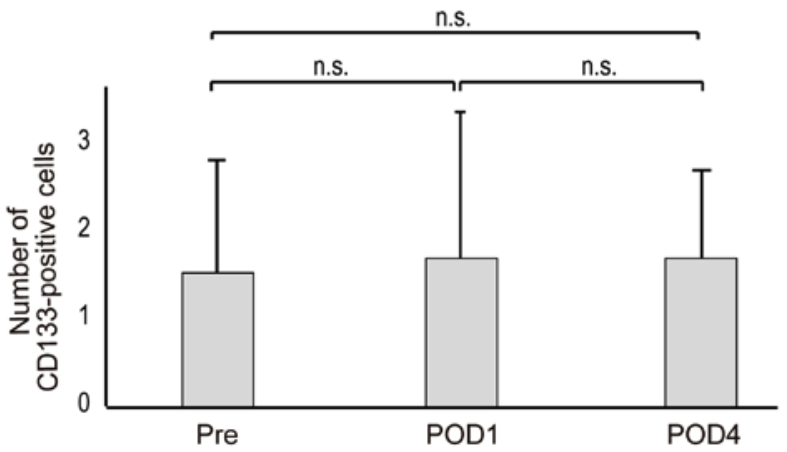

Figure 3. Images of circulating cancer stem-like cells stained with CD133. (A) CD133-positive pancreatic cancer Capan-1 cells were captured and stained with DAPI (blue), CK19 (green) and CD133 (red). A number of cells were CD133 positive (scale bar, $50 \mu \mathrm{m}$ ). (B) Representative images of the captured circulating tumor cells stained with DAPI, CK19 and CD133 in a sample obtained from patient 7 (scale bar, $50 \mu \mathrm{m}$ ). (C) The number of CD133-positive cells in the blood did not change significantly before and after SEMS placement. In seven cases (patients 7-13), CD133-positive cells were determined around stenting. The number of CD133-positive cells did not change significantly in response to stenting. SEMS, self-expandable stents; Pre, before SEMS placement; POD1 and POD4, $24 \mathrm{~h}$ and 4 days after SEMS placement, respectively; n.s., no significance.

the presence of CD133, which has also been recognized as a cancer stem-cell marker in colon cancer (23), among CTCs after SEMS placement. Because we did not observe an increase in CD133-positive CTCs after SEMS placement, even in the cases who showed significant increases in the numbers of CTCs after SEMS placement, we speculate that the temporary increase in CTCs just after the SEMS placement is not strongly related to micrometastasis potency, and most of these cells may be subject to clearance via immune surveillance, resulting in a decrease after several days. These findings may reflect the small initial number of cancer stem cells and the potential that physiological force alone does not lead to the release of cancer stem cells into the bloodstream. In fact, by our follow up data, while patient \#7 had metastatic lesions already even before stenting, patient \#8, who showed the CD133-positive cells at around SEMS placement, did not show any macroscopic metastatic lesions for one year even without any adjuvant chemotherapy after stenting. Full testing of these hypotheses requires long-term observation of the occurrence of distant metastasis after SEMS placement and the numbers of circulating cancer stem cells, examining a larger number of cases.

Currently, the only CTC detection system approved for clinical use in the United States is the automated 'CellSearch' system (24), which uses an antibody against EpCAM. Although this system has long been used as the gold standard for the detection of CTCs, it is relatively inflexible. In our study, we used our custom-made CTC detection chip, which is more flexible, as we performed immunostaining for CD133 after the capture of CTCs by antibodies against EpCAM. As aforementioned, the biological significance of CTCs may differ among cells, and we may identify cells with more malignant potential, such as cancer stem cells, by using specific antibodies against those cells. Because we can change the antibodies for cell capture in this system as needed, we can focus on biologically significant antigen-expressing cells other than EpCAM. Therefore, in the future, the capture of specific CTCs by our system and examination of relationships between such CTC features and clinical courses may provide novel insights in the field of clinical oncology.

\section{Acknowledgements}

The authors would like to thank Ms. Sayaka Ito (Department of Gastroenterology, University of Tokyo, Japan) for technical assistance.

\section{Funding}

The current study was supported by Grants-in-Aid from the Ministry of Education, Culture, Sports, Science and 
Technology of Japan (grant nos. 19H03430, 18H05024 and 17K15923), Scientific Research on Innovative Areas (grant no. 18H05024), The Yasuda Medical Foundation, the Japan Coffee Association, the Japan Foundation for Applied Enzymology and the Project for Cancer Research And Therapeutic Evolution from Japan Agency for Medical Research and Development (grant no. JP18cm0106602).

\section{Availability of data and materials}

The datasets used and/or analyzed are available from the corresponding author on reasonable request.

\section{Authors' contributions}

RI, TK and MO designed the current study and wrote the manuscript. RI, NO, TK, ET, KS and TS performed the majority of the experiments. RI, SY, RK, AN, RH and NT collected clinical samples. TO created the CTC-chip. KK designed and supervised the entire project. All authors read and approved the final manuscript.

\section{Ethics approval and consent to participate}

The study protocol was approved by the Institutional Review Board of the University of Tokyo Hospital (approval no. 11557). Written informed consent was obtained from all participating patients prior to enrollment.

\section{Patient consent for publication}

Written informed consent for publication was obtained from all participating patients.

\section{Competing interests}

The authors declare that they have no competing interests.

\section{References}

1. de Albuquerque A, Kubisch I, Stölzel U, Ernst D, Boese-Landgraf J, Breier G, Stamminger G, Fersis N and Kaul S: Prognostic and predictive value of circulating tumor cell analysis in colorectal cancer patients. J Transl Med 10: 222, 2012.

2. Ohnaga T, Shimada Y, Takata K, Obata T, Okumura T, Nagata T, Kishi H, Muraguchi A and Tsukada K: Capture of esophageal and breast cancer cells with polymeric microfluidic devices for CTC isolation. Mol Clin Oncol 4: 599-602, 2016.

3. Su DW and Nieva J: Biophysical technologies for understanding circulating tumor cell biology and metastasis. Transl Lung Cancer Res 6: 473-485, 2017.

4. Gabriel MT, Calleja LR, Chalopin A, Ory B and Heymann D: Circulating tumor cells: A review of non-EpCAM-based approaches for cell enrichment and isolation. Clin Chem 62: 571-581, 2016.

5. Chikaishi Y, Yoneda K, Ohnaga T and Tanaka F: EpCAMindependent capture of circulating tumor cells with a 'universal CTC-chip'. Oncol Rep 37: 77-82, 2017.

6. Global Burden of Disease Cancer Collaboration, Fitzmaurice C, Akinyemiju TF, Al Lami FH, Alam T, Alizadeh-Navaei R, Allen C, Alsharif U, Alvis-Guzman N, Amini E, et al: Global, regional, and national cancer incidence, mortality, years of life lost, years lived with disability, and disability-adjusted life-years for 29 cancer groups, 1990 to 2016: A systematic analysis for the global burden of disease study. JAMA Oncol 4: $1553-1568,2018$.
7. Little MW, Oakley T, Briggs JH, Sutcliffe JA, Allouni AK, Makris G, Bratby MJ, Tapping CR, Patel R, Wigham A, et al: Technical and clinical outcomes following colonic stenting: A seven-year analysis of 268 procedures. Cardiovasc Intervent Radiol 39: 1471-1478, 2016.

8. Saito S, Yoshida S, Isayama $\mathrm{H}$, Matsuzawa $\mathrm{T}$, Kuwai T, Maetani I, Shimada M, Yamada T, Tomita M, Koizumi K, et al: A prospective multicenter study on self-expandable metallic stents as a bridge to surgery for malignant colorectal obstruction in Japan: Efficacy and safety in 312 patients. Surg Endosc 30: 3976-3986, 2016.

9. Saida Y, Enomoto T, Takabayashi K, Otsuji A, Nakamura Y, Nagao J and Kusachi S: Outcome of 141 cases of self-expandable metallic stent placements for malignant and benign colorectal strictures in a single center. Surg Endosc 25: 1748-1752, 2011

10. Maruthachalam K, Lash GE, Shenton BK and Horgan AF: Tumour cell dissemination following endoscopic stent insertion. Br J Surg 94: 1151-1154, 2007.

11. Sabbagh C, Browet F, Diouf M, Cosse C, Brehant O, Bartoli E, Mauvais F, Chauffert B, Dupas JL, Nguyen-Khac E and Regimbeau JM: Is stenting as 'a bridge to surgery' an oncologically safe strategy for the management of acute, left-sided, malignant, colonic obstruction? A comparative study with a propensity score analysis. Ann Surg 258: 107-115, 2013.

12. van den Berg MW, Sloothaak DA, Dijkgraaf MG, van der Zaag ES, Bemelman WA, Tanis PJ, Bosker RJ, Fockens P, ter Borg $\mathrm{F}$ and van Hooft JE: Bridge-to-surgery stent placement versus emergency surgery for acute malignant colonic obstruction. Br J Surg 101: 867-873, 2014.

13. Yamashita S, Tanemura M, Sawada G, Moon J, Shimizu Y, Yamaguchi T, Kuwai T, Urata Y, Kuraoka K, Hatanaka N, et al: Impact of endoscopic stent insertion on detection of viable circulating tumor cells from obstructive colorectal cancer. Oncol Lett 15: 400-406, 2018.

14. Takahashi G, Yamada T, Iwai T, Takeda K, Koizumi M, Shinji S and Uchida E: Oncological assessment of stent placement for obstructive colorectal cancer from circulating cell-free DNA and circulating tumor DNA dynamics. Ann Surg Oncol 25: 737-744, 2018.

15. de Sousa e Melo F, Kurtova AV, Harnoss JM, Kljavin N, Hoeck JD, Hung J, Anderson JE, Storm EE, Modrusan Z, Koeppen $\mathrm{H}$, et al: A distinct role for Lgr5+ stem cells in primary and metastatic colon cancer. Nature 543: 676-680, 2017.

16. Chen S, Song X, Chen Z, Li X, Li M, Liu H and Li J: CD133 expression and the prognosis of colorectal cancer: A systematic review and meta-analysis. PLoS One 8: e56380, 2013.

17. Nagata H, Ishihara S, Kishikawa J, Sonoda H, Murono K, Emoto S, Kaneko M, Sasaki K, Otani K, Nishikawa T, et al: CD133 expression predicts post-operative recurrence in patients with colon cancer with peritoneal metastasis. Int J Oncol 52: 721-732, 2018.

18. Ohnaga T, Shimada Y, Moriyama M, Kishi H, Obata T, Takata K, Okumura T, Nagata T, Muraguchi A and Tsukada K: Polymeric microfluidic devices exhibiting sufficient capture of cancer cell line for isolation of circulating tumor cells. Biomed Microdevices 15: 611-616, 2013.

19. Cima I, Kong SL, Sengupta D, Tan IB, Phyo WM, Lee D, Hu M, Iliescu C, Alexander I, Goh WL, et al: Tumor-derived circulating endothelial cell clusters in colorectal cancer. Sci Transl Med 8: 345ra89, 2016.

20. Tilney HS, Lovegrove RE, Purkayastha S, Sains PS, Weston-Petrides GK, Darzi AW, Tekkis PP and Heriot AG: Comparison of colonic stenting and open surgery for malignant large bowel obstruction. Surg Endosc 21: 225-233, 2007.

21. Arezzo A, Balague C, Targarona E, Borghi F, Giraudo G, Ghezzo L, Arroyo A, Sola-Vera J, De Paolis P, Bossotti M, et al: Colonic stenting as a bridge to surgery versus emergency surgery for malignant colonic obstruction: Results of a multicentre randomised controlled trial (ESCO trial). Surg Endosc 31: 3297-3305, 2017.

22. Schild T, Low V, Blenis J and Gomes AP: Unique metabolic adaptations dictate distal organ-specific metastatic colonization. Cancer Cell 33: 347-354, 2018.

23. Zeki SS, Graham TA and Wright NA: Stem cells and their implications for colorectal cancer. Nat Rev Gastroenterol Hepatol 8: 90-100, 2011.

24. Riethdorf S, Fritsche H, Müller V, Rau T, Schindlbeck C, Rack B, Janni W, Coith C, Beck K, Jänicke F, et al: Detection of circulating tumor cells in peripheral blood of patients with metastatic breast cancer: A validation study of the CellSearch system. Clin Cancer Res 13: 920-928, 2007.

This work is licensed under a Creative Commons Attribution-NonCommercial-NoDerivatives 4.0 International (CC BY-NC-ND 4.0) License. 